\title{
APPROXIMATE ANALYTICAL SOLUTION TO THE CATTANEO HEAT CONDUCTION MODEL WITH VARIOUS LASER SOURCES
}

\author{
Rahmatullah Ibrahim Nuruddeen \\ Department of Mathematics, Federal University Dutse \\ 7156 Dutse, Jigawa State, Nigeria \\ rahmatullah.n@fud.edu.ng
}

Received: 1 August 2021; Accepted: 25 October 2021

\begin{abstract}
The present manuscript investigates the role being played by various laser short heating sources in a conduction process of a metallic substrate. The Cattaneo heat conduction model is considered in favour of its finiteness of conduction speed. The analytical solutions for the temperature fields are determined via the application of the Laplace integral transform. Finally, we sought a numerical Laplace inversion scheme where the analytical inversion failed and graphically examined the significance of the heating parameters on the temperature fields.
\end{abstract}

MSC 2010: 80A20, 65R10, 35A22, 35L15

Keywords: Cattaneo model, heat conduction, laser heating, Laplace transform, numerical inversion.

\section{Introduction}

Heat conduction processes have been extensively studied in the literature in favor of their vast applications in many science and engineering areas. These studies were mostly based upon the classical Fourier's model [1] that accounts for an infinite speed of heat transfer. This defect alongside its unsuitability in predicting low-temperature scenarios and others resulted in many models being proposed including, in particular, the famous hyperbolic Cattaneo heat conduction model [2]. The Cattaneo model has been able to account for a finite speed and adequately modeled heating problems driven by external sources with short durations like laser short pulses among others, see $[3,4]$ and the references therein. The laser short pulse process is a burning area of research due to the current innovative and technological advancements. Metallic substrate surfaces are treated with laser pulses for more rigidity and other applications $[5,6]$.

Moreover, the determination of temperature fields in various media with and without external heating sources remains an open problem with regards to various structural configurations and, on the other hand, the emerging mathematical techniques. In line with this, various approximate and analytical methods have been employed 
in the last decades to scrutinize different forms of heat conduction models. For instance, Nuruddeen and Zaman [7, 8] and Al-Duhaim et al. [9] analyzed heating and thermal stress scenarios with mixed boundary conditions, respectively, using the Wiener-Hopf technique. Yan et al. [10] proposed and utilized a novel series method to examine the fractional diffusion equation; while Al-Khaled and Momani [11] investigated the diffusion-wave equation with a fractional-order via the use of the approximate decomposition approach. Other related studies include the investigation of the nonlinear heat diffusion model, comparative study of the Caputo and conformable fractional-order derivatives in relation to the heat conduction equation, tackling heat equation with nonlocal conditions, a method for the fractional conduction models, and the treatment of the nonlinear conduction process were all studied using methods based on the Adomian's approach [12-18]. Other approaches are comprised of the Lie's symmetry method for the reduction of the heat conduction equation [19] and the eigenfunctions expansion approach to a layered conduction slab [20] to mention a few.

However, the present study is motivated by the fact that the Cattaneo heat conduction equation possesses finiteness of conduction speed alongside its ability to model low-temperature scenarios like laser short heating sources. Thus, we investigate in this manuscript the role of three different laser short heating sources on a conducting half-plane metallic substrate modeled using the Cattaneo heat conduction equation. The analytical solutions for the temperature fields will be sought using the Laplace integral transform [21, 22]; see also [23, 26] for other relevant methodologies. Equally, we will utilize the numerical Laplace inversion scheme by Abate and Valkó [27] where the analytical inversion fails and goes ahead with determination of the respective temperature fields. Furthermore, the present paper takes the following organization: Section 2 gives some basics about the heating process, together with the method to be utilized. In Section 3, we give the formulation of the aiming problem to tackle in this study. Section 4 gives the application of the outlined method on the formulated problem for the determination of the temperature fields. Section 5 presents the obtained results graphically and makes some discussions; while Section 6 gives some concluding remarks.

\section{Preliminaries and methods}

This section outlines some basics about the heat conduction process, together with the method to be utilized; specifically, the Laplace integral transform and its inverse.

\subsection{Heat conduction equation}

Here, we consider the two famous heat conduction equations based on the Fourier's law and the modified constitutive equation by Cattaneo. 


\subsubsection{Fourier's heat conduction equation}

The classical one-dimensional parabolic heat conduction equation based on the Fourier's law in the presence of a heating source function $f(x, t)$ reads as follows [1]

$$
B \frac{\partial w}{\partial t}=\frac{\partial^{2} w}{\partial x^{2}}+f(x, t)
$$

where $w=w(x, t)$ denotes the temperature field $[\mathrm{K}]$ in space $x$ and time $t$ variables (measured in $m$ and $s$, respectively), and $B=\frac{\rho c_{p}}{k}\left[\mathrm{~s} / \mathrm{m}^{2}\right]$ with $\rho-$ the density $\left[\mathrm{kg} / \mathrm{m}^{3}\right], c_{p}-$ the specific heat capacity $[\mathrm{J} /(\mathrm{kg} \mathrm{K})]$ and $k-$ the thermal diffusivity of the medium $[\mathrm{W} /(\mathrm{m} \mathrm{K})]$, correspondingly.

\subsubsection{Cattaneo heat conduction equation}

The one-dimensional hyperbolic heat conduction equation that accounts for a finite speed based on the modified constitutive equation by Cattaneo in the presence of a heating source function $f(x, t)$ reads as follows [2-4]

$$
A \frac{\partial^{2} w}{\partial t^{2}}+B \frac{\partial w}{\partial t}=\frac{\partial^{2} w}{\partial x^{2}}+f(x, t)
$$

where $w=w(x, t)$ denotes the temperature field $[\mathrm{K}]$ in space $x$ and time $t$ variables (measured in $m$ and $s$, respectively), $A=\frac{\rho c_{p} \tau}{k}\left[\mathrm{~s}^{2} / \mathrm{m}^{2}\right]$, and $B=\frac{\rho c_{p}}{k}\left[\mathrm{~s} / \mathrm{m}^{2}\right]$ with $\tau$ - the time relaxation $[\mathrm{s}], \rho$ - the density $\left[\mathrm{kg} / \mathrm{m}^{3}\right], c_{p}$ - the specific heat capacity $[\mathrm{J} /(\mathrm{kgK})]$ and $k$ - the thermal diffusivity of the medium $[\mathrm{W} /(\mathrm{mK})]$, correspondingly.

\subsection{Methodology}

The present study employs the Laplace integral transform as the methodology of the study. We therefore give more about the details of this method in this subsection.

\subsubsection{Laplace transform}

The definition of the classical Laplace integral transform [21, 22] and its corresponding inverse transform will respectively be utilized in this study. What's more, $s$ will denote the Laplace transform's parameter throughout the manuscript.

\subsubsection{Numerical inverse Laplace transform}

Furthermore, in a situation where by the analytical inversion of the Laplace transform is not possible, we will resort to using the powerful numerical Laplace inversion 
scheme by Abate and Valkó [27]. In [27], the numerical Laplace inversion scheme was proposed at a given time point, say $t_{0}$, and is implementable on the Mathematica software via the method's inversion package.

\section{Problem formulation}

In this section, we give the statement of the problem with regards to the temperature determination we are aiming to tackle in this study and, the three laser source functions to examine.

\subsection{Statement of the problem}

We consider a half-space metallic substrate lying in $0 \leq x<\infty$ and driven by a laser pulse heating source $f(x, t)$. The temperature distribution in the substrate is thus governed by the one-dimensional hyperbolic Cattaneo heat conduction equation given in equation (2). We also prescribe the following initial conditions

$$
\begin{aligned}
w(x, t) & =u_{0}, \quad \text { at } \quad t=0, \\
\frac{\partial w}{\partial t}(x, t) & =0, \quad \text { at } \quad t=0,
\end{aligned}
$$

where $u_{0}$ is a prescribed constant temperature of which the material is kept before the heating process begins. What's more, we also consider the following boundary conditions

$$
\begin{gathered}
\frac{\partial w}{\partial x}(x, t)=0, \quad \text { on } \quad x=0, \\
\lim _{x \rightarrow \infty} w(x, t)=0 .
\end{gathered}
$$

In the above boundary conditions, the heat flux is assumed to be zero at $x=0$; while the temperature varnishes at a very large depth.

\subsection{Laser heating sources}

With regards to the laser source function $f(x, t)$ in equation (2), we consider three different laser heating sources here that will later be analyzed. The heating sources are as follows:

- Source I: the laser short-pulse heating source by Yibas et al. [5] given by

$$
f(x, t)=I_{0} \eta e^{-\eta x} .
$$


- Source II: the laser short-pulse heating source by Yibas et al. [6] given by

$$
f(x, t)=I_{0} \eta e^{-\eta x} e^{-\gamma t} .
$$

- Source III: the laser short-pulse heating source of volumetric nature by Al Duhaim et al. [4] of the form

$$
f(x, t)=I_{0} \eta e^{-\eta x}\left(e^{-\gamma t}-e^{-\beta t}\right) .
$$

Also, in the above equations (5), (6) and (7), $I_{0}$ is the laser peak power intensity $\left[\mathrm{W} / \mathrm{m}^{2}\right], \eta$ is the absorption coefficient $[1 / \mathrm{m}]$; while $\beta$ and $\gamma$ are the laser pulse parameters $[1 / \mathrm{s}]$, respectively.

\section{Temperature determination}

This section employs the Laplace integral transform method earlier outlined to tackle the formulated problem of the heat conduction process via the Cattaneo model given in equation (2) and driven by laser source functions as expressed in equations (5)-(7).

\subsection{Temperature in the presence of source $I$}

Considering the hyperbolic Cattaneo model given in equation (2) in the presence of the laser heating Source I given in equation (5), the explicit model becomes

$$
A \frac{\partial^{2} w}{\partial t^{2}}+B \frac{\partial w}{\partial t}=\frac{\partial^{2} w}{\partial x^{2}}+I_{0} \eta e^{-\eta x}
$$

Now, on taking the Laplace transform in $t$ of equation (8), the equation after utilizing the initial conditions given in equation (3) reduces to the following second-order ordinary differential equations

$$
\frac{\partial^{2} w^{*}}{\partial x^{2}}-r^{2} w^{*}=-u_{0}(A s+B)-\frac{\eta I_{0} e^{-\eta x}}{s},
$$

where

$$
r=\sqrt{A s^{2}+B s}
$$

Similarly, the transformed boundary conditions with respect to the Laplace transform are

$$
\begin{gathered}
\frac{\partial w^{*}(x, s)}{\partial x}=0, \quad \text { on } \quad x=0, \\
\lim _{x \rightarrow \infty} w^{*}(x, s)=0 .
\end{gathered}
$$


Therefore, the ordinary differential equation given in equation (9) admits the following analytic exact solution

$$
w^{*}(x, s)=\frac{u_{0}(A s+B)}{r^{2}}+\frac{\eta I_{0} e^{-\eta x}}{s\left(r^{2}-\eta^{2}\right)}+c_{1} e^{r x}+c_{2} e^{-r x},
$$

where $c_{1}$ and $c_{2}$ are constants to be determined via the given boundary conditions. Furthermore, on applying the second transformed condition in equation (10), the obtained solution in the above equation further reduces to

$$
w^{*}(x, s)=\frac{u_{0}(A s+B)}{r^{2}}+\frac{\eta I_{0} e^{-\eta x}}{s\left(r^{2}-\eta^{2}\right)}+c_{2} e^{-r x} .
$$

Moreover, the complete solution after utilizing the first transformed condition in equation (10) becomes

$$
w^{*}(x, s)=\frac{u_{0}(A s+B)}{r^{2}}+\frac{\eta I_{0} e^{-\eta x}}{s\left(r^{2}-\eta^{2}\right)}-\frac{\eta^{2} I_{0} e^{-r x}}{r s\left(r^{2}-\eta^{2}\right)} .
$$

Therefore, having determined the unknowns, we then go back to equation (13) to invert the solution back to its original domain, that is, from $(x, s) \rightarrow(x, t)$. To do this, we apply the inverse Laplace transform to equation (13) to obtain

$$
\begin{aligned}
w(x, t) & =u_{0}+\frac{I_{0} \lambda_{1}(t) e^{-\eta x-\frac{t\left(\sqrt{4 A \eta^{2}+B^{2}}+B\right)}{2 A}}}{2 \eta \sqrt{4 A \eta^{2}+B^{2}}} \\
& -\frac{\eta^{2} I_{0}}{2 \pi i} \int_{c-i \infty}^{c+i \infty}\left(\frac{e^{-x \sqrt{A s^{2}+B s}}}{s \sqrt{A s^{2}+B s}\left(A s^{2}+B s-\eta^{2}\right)}\right) e^{s t} d s,
\end{aligned}
$$

where $\lambda_{1}(t)$ in the above equation is given by

$$
\lambda_{1}(t)=B\left(e^{\frac{t \sqrt{4 A \eta^{2}+B^{2}}}{A}}-1\right)+\sqrt{4 A \eta^{2}+B^{2}}\left(e^{\frac{t \sqrt{4 A \eta^{2}+B^{2}}}{A}}-2 e^{\frac{t\left(\sqrt{4 A \eta^{2}+B^{2}}+B\right)}{2 A}}+1\right) .
$$

Equation (14) is the overall solution of the temperature field in the presence of laser Source I. Also, since the third part appearing under the Laplace inversion integral cannot be evaluated analytically, we resort to using the numerical Laplace inversion scheme by Abate and Valkó [27]. In this case, we report the analytically approximated solution in Figure 1a,b at different heating time levels.

\subsection{Temperature in the presence of source II}

Considering the hyperbolic Cattaneo model given in equation (2) in the presence of the laser heating Source II given in equation (6), the explicit model reads 


$$
A \frac{\partial^{2} w}{\partial t^{2}}+B \frac{\partial w}{\partial t}=\frac{\partial^{2} w}{\partial x^{2}}+I_{0} \eta e^{-\eta x} e^{-\gamma t}
$$

Now, taking the Laplace transform in $t$ of equation (16), the equation after utilizing the initial conditions given in equation (3) reduces to the following second-order ordinary differential equation

$$
\frac{\partial^{2} w^{*}}{\partial x^{2}}-r^{2} w^{*}=-u_{0}(A s+B)-\frac{\eta I_{0} e^{-\eta x}}{s+\gamma},
$$

where

$$
r=\sqrt{A s^{2}+B s}
$$

Thus, without loss of generality, we proceed as in the preceded case to obtain the following temperature field

$$
\begin{aligned}
w(x, t)=u_{0} & +\frac{\eta I_{0} \lambda_{2}(t)}{2 \sqrt{4 A \eta^{2}+B^{2}}\left(\gamma(B-A \gamma)+\eta^{2}\right)} e^{-\eta x-\frac{t\left(\sqrt{4 A \eta^{2}+B^{2}}+B\right)}{2 A}} \\
& -\frac{\eta^{2} I_{0}}{2 \pi i} \int_{c-i \infty}^{c+i \infty}\left(\frac{\left.e^{x(-\sqrt{s(A s+B)}}\right)}{(\gamma+s) \sqrt{s(A s+B)}\left(s(A s+B)-\eta^{2}\right)}\right) e^{s t} d s,
\end{aligned}
$$

where $\lambda_{2}(t)$ in the above equation is given by

$$
\begin{aligned}
& \lambda_{2}(t)=-2 A \gamma\left(e^{\frac{t \sqrt{4 A \eta^{2}+B^{2}}}{A}}-1\right)+B\left(e^{\frac{t \sqrt{4 A \eta^{2}+B^{2}}}{A}}-1\right)+ \\
& \sqrt{4 A \eta^{2}+B^{2}}\left(-2 e^{\frac{t\left(\sqrt{4 A \eta^{2}+B^{2}}-2 A \gamma+B\right.}{2 A}}+e^{\frac{t \sqrt{4 A \eta^{2}+B^{2}}}{A}}+1\right) .
\end{aligned}
$$

Equation (18) is the overall solution of the temperature field in the presence of laser Source II. Again, since the third part appearing under the Laplace inversion integral cannot be evaluated analytically, we resort to using the numerical Laplace inversion scheme by Abate and Valkó [27]. We therefore report the analytically approximated results in Figure 2a-c at different heating time levels.

\subsection{Temperature in the presence of source III}

Considering the hyperbolic Cattaneo model given in equation (2) in the presence of the laser heating Source III given in equation (7), the explicit model is expressed as follows

$$
A \frac{\partial^{2} w}{\partial t^{2}}+B \frac{\partial w}{\partial t}=\frac{\partial^{2} w}{\partial x^{2}}+I_{0} \eta e^{-\eta x}\left(e^{-\gamma t}-e^{-\beta t}\right)
$$


Also, on taking the Laplace transform in $t$ of equation (20), the equation after utilizing the given initial conditions in equation (3) reduces to the following second-order ordinary differential equation

$$
\frac{\partial^{2} w^{*}}{\partial x^{2}}-r^{2} w^{*}=-u_{0}(A s+B)-\eta I_{0}\left(\frac{1}{\gamma+s}-\frac{1}{\beta+s}\right) e^{-\eta x},
$$

where

$$
r=\sqrt{A s^{2}+B s}
$$

Also, without loss of generality, we proceed as in the above case to obtain the following temperature field

$$
w(x, t)=u_{0}+\frac{1}{2 \pi i} \int_{c-i \infty}^{c+i \infty} \lambda_{3}(s) e^{s t} d s+\frac{1}{2 \pi i} \int_{c-i \infty}^{c+i \infty} \lambda_{4}(s) e^{s t} d s,
$$

where $\lambda_{3}(t)$ are $\lambda_{4}(t)$ are expressed as follows

$$
\begin{gathered}
\lambda_{3}(s)=\frac{\eta^{2}(\gamma-\beta) I_{0}}{(\beta+s)(\gamma+s) \sqrt{A s^{2}+B s}\left(\sqrt{A s^{2}+B s}-\eta\right)\left(\sqrt{A s^{2}+B s}+\eta\right)} e^{-x \sqrt{A s^{2}+B s}} \\
\lambda_{4}(s)=\frac{\eta(\gamma-\beta) I_{0}}{(\beta+s)(\gamma+s)\left(\eta-\sqrt{A s^{2}+B s}\right)\left(\sqrt{A s^{2}+B s}+\eta\right)} e^{-\eta x}
\end{gathered}
$$

Again, equation (22) is the overall solution of the temperature field in the presence of laser Source III. Furthermore, since the second and third parts appearing under the Laplace inversion integral cannot be evaluated analytically, we resort to using the numerical Laplace inversion scheme by Abate and Valkó [27]. We therefore report the analytically approximated results in Figure 3a-c at different heating time levels.

\section{Results and discussion}

This study investigates the significance of the three laser short heating sources given in equations (5)-(7) on a conducting metallic substrate. The hyperbolic Cattaneo heat conduction model is considered. The solutions for the temperature fields are obtain analytically using the Laplace integral transform method. Also, we utilize the numerical Laplace inversion scheme by Abate and Valkó [27] where the analytical inversion process fails. Interesting graphical depictions to visualize the obtained results are reported in Figures 1-3. Furthermore, we consider the substrate parameters as $A=0.8 \mathrm{~s}^{2} \mathrm{~m}^{-2}, B=1 \mathrm{sm}^{-2}$, and the laser parameters $I_{0}=1 \mathrm{Wm}^{-2}$, and we consider the rest to be less than or equal to 1 . We also fix the initial temperature to be 
$u_{0}=1 \mathrm{~K}$. With this, we then study the variational effects of ther other laser heating parameters on the temperature fields at different heating time levels.

Figure 1a,b shows the temperature variations driven by the laser Source I. In both (a) and (b), the temperature distributions increase with an increase in time $t$ and eventually decays out along the substrate's depth. It is noted that a decrease in $\eta$ results in a decrease of the decaying rate as can be seen in Figure $1 \mathrm{~b}$ when $\eta=0.25 \mathrm{~m}^{-1}$.

Figure 2a-c shows the temperature variations driven by the laser Source II. The same interpretation of Figure 1a,b goes to Figure 2a,b when $\gamma=1 \mathrm{~s}^{-1}$ at $\eta=1 \mathrm{~m}^{-1}$ and $\eta=0.25 \mathrm{~m}^{-1}$, respectively, but with a slight difference of the temperature rise. However, when $\gamma$ decreases to 0.35 as shown in Figure 2c, the temperature distribution begins to rise at the same time with an increase in the decaying rate in comparison to Figure $2 \mathrm{~b}$ when $\eta=0.25 \mathrm{~m}^{-1}$ and $\gamma=1 \mathrm{~s}^{-1}$.

Figure $3 \mathrm{a}-\mathrm{c}$ shows the temperature variations driven by the laser Source III. Again here, the same interpretation of Figure 1a,b applies to Figure 3a,b but with a slight difference of the temperature rise. This, in fact, means the presence of more laser parameters shrinks the rate at which the temperature rises. This effect can be noted in all the Sub-figures in Source III. Now, with regards to the Figure 3c, a complete opposite behaviour is observed. This behaviour is equivalent to the behaviour of the thermal stress of the same laser source examined by Al-Duhaim et al. [3] via the Laplace and Fourier cosine integral transforms. This in fact is caused by a simultaneous increase of any of the two parameters. Also, in this case, the temperature approaches the constant initial temperature $u_{0}=1 \mathrm{~K}$ even at a higher depth. This indeed contradicts the boundedness boundary condition prescribed at the higher depth of the substrate in the formulation; thus, this situation is discarded.

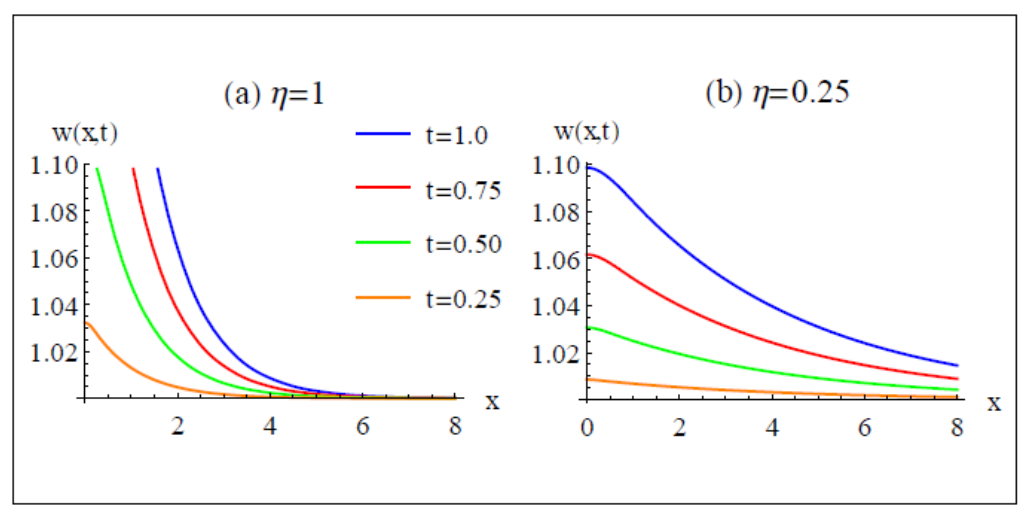

Fig. 1. Two-dimensional visualization of the temperature field determined in equation (14) under Source I at different heating time levels when: a) $\eta=1$, and b) $\eta=0.25$ 


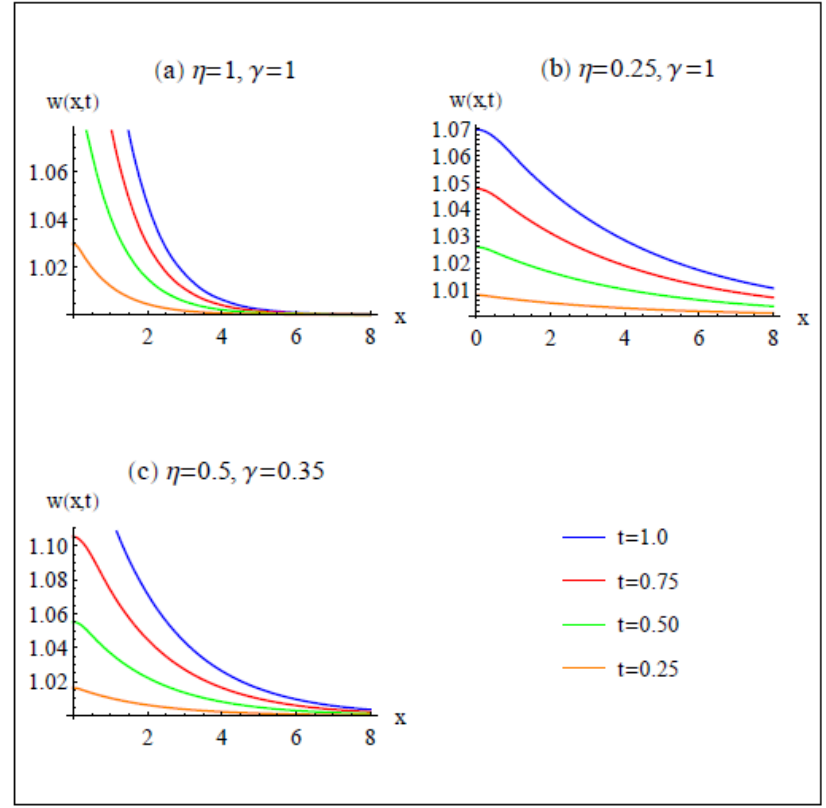

Fig. 2. Two-dimensional visualization of the temperature field determined in equation (18) under Source II at different heating time levels when: a) $\eta=1, \gamma=1$, b) $\eta=0.25, \gamma=1$, and c) $\eta=0.5, \gamma=0.35$

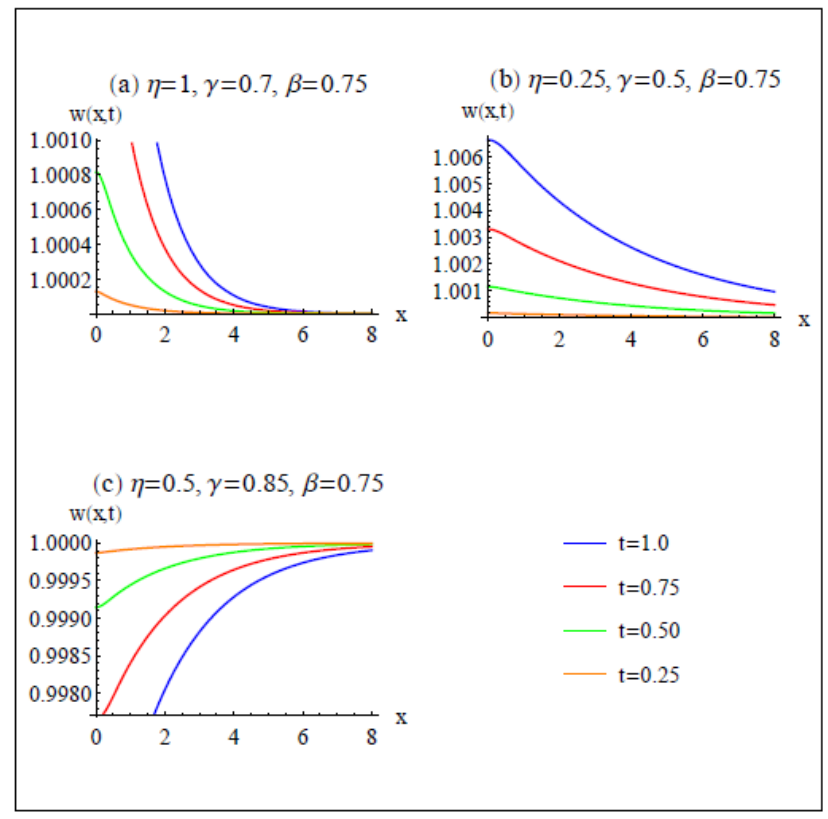

Fig. 3. Two-dimensional visualization of the temperature field determined in equation (22) under Source III at different heating time levels when: a) $\eta=1, \gamma=0.7, \beta=0.75$,

b) $\eta=0.25, \gamma=0.5, \beta=0.75$, and (c) $\eta=0.5, \gamma=0.85, \beta=0.75$. 


\section{Conclusion}

In conclusion, the present manuscript investigated the significance of three different laser short heating sources on a conducting metallic substrate. The hyperbolic Cattaneo heat conduction model was considered for its finiteness of conduction speed. The analytical solutions for the temperature fields were sought analytically using the Laplace integral transform method. Moreover, we utilized the numerical Laplace inversion scheme by Abate and Valkó [27] where the analytical inversion failed and further went ahead with determination of the respective temperature fields. Finally, certain interesting graphical depictions were supplied to visualize the obtained results, alongside noting the variational effects of the laser short pulse heating on the resulting temperature fields.

\section{References}

[1] Carslaw, H., \& Jeager, J.C. (1959). The Conduction of Heat in Solids. 2nd edition, Oxford: Clarendon Press.

[2] Qi, H.T., Xu., H.Y., \& Guo, X.W. (2013). The Cattaneo-type time fractional heat conduction equation for laser heating. Computers and Mathematics with Applications, 66, 824-831.

[3] Al-Duhaim, H.R., Yilbas, B.S., \& Zaman, F.D. (2016). Determination of temperature distribution and thermal stress for the hyperbolic heat conduction equation due to laser short pulse heating. Lasers in Engineering, 35, 275-301.

[4] Al-Duhaim, H.R., Yilbas, B.S., \& Zaman, F.D. (2017). Hyperbolic nature of heat conduction for short pulse laser irradiation of solid surfaces: analytical solution for the thermal stress field. Lasers in Engineering, 36, 331-353.

[5] Yilbas, B.S., Akhtar, S., \& Shuja, S.Z. (2013). Laser Forming and Welding. Springer.

[6] Yilbas, B.S., Al-Dweik, A.Y., \& Bin Mansour, S. (2011). Analytical solution of hyperbolic heat conduction equation in relation to laser short-pulse heating. Physica B, 406, 1550-1555.

[7] Nuruddeen, R.I., \& Zaman, F.D. (2016). Heat conduction of a circular hollow cylinder amidst mixed boundary conditions. International Journal of Scientic Engineering and Technology, 5(1), 18-22.

[8] Nuruddeen, R.I., \& Zaman, F.D. (2016). Temperature distribution in a circular cylinder with general mixed boundary conditions. Journal of Multidisciplinary Engineering Science and Technology, 3(1), 3653-3658.

[9] Al-Duhaim, H.R., Nuruddeen, R.I., \& Zaman, F.D. (2015). Thermal stress in a half-space with mixed boundary conditions due to time dependent heat source. IOSR Journal of Mathematics, 11(6), 19-25.

[10] Yan, S.P., Zhong, W.P., \& Yang, X.J. (2016). A novel series method for fractional diffusion equation within Caputo fractional derivative. Thermal Science, 20(3), S695-S699.

[11] Al-Khaled, K., \& Momani, S. (2005). An approximate solution for a fractional diffusion-wave equation using the decomposition method. Applied Mathematics and Computation, 2(15), 473-483.

[12] Ray, S.S., \& Bera, R.K. (2006). Analytical solution of a fractional diffusion equation by Adomian decomposition method. Applied Mathematics and Computation, 174, 329-336.

[13] Bokhari, A.H., et al. (2009). Adomian decomposition method for a nonlinear heat equation with temperature dependent thermal properties. Mathematical Problems in Engineering. 
[14] Bokhari, A.H., et al. (2009). Solution of heat equation with nonlocal boundary conditions. International Journal of Mathematics and Computation, 3(J09), 100-113.

[15] Nuruddeen, R.I., Zaman, F.D., \& Zakariya, Y.F. (2019). Analysing the fractional heat diffusion equation solution in comparison with the new fractional derivative by decomposition method. Malaya Journal of Matematik, 7(2), 213-222.

[16] Nuruddeen, R.I., \& Garba, B.D. (2018). Analytical technique for (2+1) fractional diffusion equation with nonlocal boundary conditions. Open Journal of Mathematics Science, 2(1), 287-300.

[17] Al Qarni, A., et al. (2019). Bright optical solitons for Lakshmanan-Porsezian-Daniel model with spatio-temporal dispersion by improved Adomian decomposition method. Optik, 181, 891-897.

[18] Nuruddeen, R.I., et al. (2018). A review of the integral transforms-based decomposition methods and their applications in solving nonlinear PDEs. Palestine Journal od Mathematics, 7, 262-280.

[19] Ahmad, A., et al. (2008). Symmetry classifications and reductions of some classes of (2+1)-nonlinear heat equation. Journal of Mathematical Analysis and Applications, 339(1), 175-181.

[20] Masood, K., \& Zaman, F.D. (2004). Initial inverse problem in a two-layer heat conduction model. The Arabian Journal for Science and Engineering, 29(1B), 1-12.

[21] Laplace, P.S. (2820). Theorie Analytique des Probabilities. Paris: Lerch.

[22] Debnath, L., \& Bhatta, D. (2007). Integral Transforms and their Applications. 2nd ed. Boca Raton: Taylor \& Francis Group, LLC.

[23] Kukla, S., \& Siedlecka, U. (2020). Time-fractional heat conduction in a finite composite cylinder with heat source. Journal of Applied Mathematics and Computational Mechanics, 19(2), 85-94.

[24] Jan, R., \& Jan, A. (2017). MSGDTM for solution of fractional order dengue disease model. International Journal of Science and Research, 6(3), 1140-1144.

[25] Alharbi, F.M., et al. (2021). Bioconvection due to gyrotactic microorganisms in couple stress hybrid nanofluid laminar mixed convection incompressible flow with magnetic nanoparticles and chemical reaction as carrier for targeted drug delivery through porous stretching sheet. Molecules, 26(13) 3954.

[26] Jan, R., et al. (2021). The investigation of the fractional-view dynamics of helmholtz equations within Caputo Operator. CMC-Computers Materials \& Continua, 68(3), 3185-3201.

[27] Abate, J., \& Valkó, P.P. (2004). Multi-precision Laplace transform inversion. Numerical Methods in Engineering, 60(5), 979-993. 